\title{
Mariátegui y Lipschutz. Influencias conceptuales para una historia andina en clave marxista (Chile, 1970)
}

\author{
Mariátegui and Lipschutz. Conceptual influences for an Andean history in Marxist key \\ (Chile, 1970)
}

Carlos María Chiappe*

\begin{abstract}
Resumen: Se analiza la influencia del pensamiento de los indigenistas José Carlos Mariátegui y Alejandro Lipschutz en un conjunto de investigadores marxistas interesados por problemáticas del Norte de Chile. Se procura entender su incidencia en el abordaje de temas como la desintegración étnica y las autonomías cultural y política y en la construcción académica sobre el pasado, el presente y el futuro de los pueblos andinos de la zona.
\end{abstract}

Palabras clave: marxismo, indigenismo, estudios andinos, Chile, 1970

\begin{abstract}
In this article we analyze the influence of José Carlos Mariátegui and Alejandro Lipschutz on a marxist's researchers network interested in isssues from Northern Chile. This is done with the aim of understanding its impact on issues such as ethnic disintegration and cultural and political autonomy and the academic construction of the past, present and future of the Andean peoples of the area.
\end{abstract}

Key words: Marxism, Indigenism, Andean studies, Chile, 1970

Recibido: 21 diciembre 2018 Aceptado: 6 marzo 2019

\section{Introducción}

El presente trabajo analiza la influencia de Mariátegui y Lipschutz en los desarrollos teóricos de una red de investigadores marxistas interesados en las problemáticas de las poblaciones originarias del norte de Chile. El referido grupo se conformó durante la década de 1960 en torno a la articulación entre militancia política y proyectos de investigación en el campo de los estudios andinos. En orden de aquilatar esta influencia, analizaré en el primer apartado los aportes de Lipschutz, principal figura del indigenismo chileno. En el segundo apartado realizaré una breve semblanza de los investigadores incluidos en la red, de sus perfiles académicos y de sus relaciones con diversas figuras, entre ellas el mismo Lipschutz. En el tercer apartado estudiaré la influencia de los dos indigenistas citados en la producción textual originada en ocasión del I Congreso del Hombre Andino (Arica, Iquique y Antofagasta, 1973). Se trata, en primer lugar, de la conferencia de apertura del Congreso, autoría de Lipschutz, y del Informe de tema del Simposio 4, realizado por la Comisión Organizadora, la cual estaba conformada por la red de cientistas

\footnotetext{
* Argentino. Dr. en Antropología. Universidad Católica del Norte, Instituto de Arqueología y Antropología. San Pedro de Atacama, Chile carlosmariachiappe@gmail.com
} 
en marras. En este informe es dable de observar una marcada influencia teórica de las proposiciones de Mariátegui. En las conclusiones, el análisis de ambos textos me permitirá explicitar la forma en la que era entendido el llamado "problema indígena", los acuerdos y desacuerdos en torno al mismo y su incidencia en temas como la desintegración étnica y las autonomías cultural y política y el pasado, presente y futuro de los pueblos andinos.

\section{Lipschutz y la transculturación}

\section{El concepto de transculturación}

En 2017 asistí en Buenos Aires a una conferencia del Dr. Eduardo Devés-Valdés. ${ }^{1}$ El tema de la misma llevó a preguntarme que aportes a la teoría social había encontrado yo -en el marco de mis investigaciones dedicadas a la producción y circulación del conocimiento científico- desde lo que se denomina pensamiento periférico. ${ }^{2}$ Inmediatamente vino a mi memoria el concepto de transculturación y de allí surgió la idea de este artículo.

El concepto de transculturación fue acuñado por el cubano Fernando Ortiz para discutir conceptos provenientes de la antropología de las academias centrales -básicamente la estadounidense y las del occidente de Europa- apareciendo por primera vez en Contrapunteo cubano del tabaco y el azúcar. ${ }^{3}$ En la Introducción, Bronisław Malinowski, renovador metodológico de la antropología social británica, recuerda: ${ }^{4}$

[...] me encontré personalmente con Fernando Ortiz durante mi primera visita a La Habana, en noviembre del año 1929 [...] El doctor Ortiz me dijo entonces que en su próximo libro iba a introducir un nuevo vocablo técnico, el termino transculturación, para reemplazar varias expresiones corrientes, tales como "cambio cultural", "aculturación", "difusión", "migración u ósmosis de la cultura" y otras análogas que él consideraba como de sentido imperfectamente expresivo. Mi respuesta desde el primer momento fue de entusiasta acogida para ese neologismo. Y le prometí a su autor que yo me apropiaría de la nueva expresión, reconociendo su paternidad, para usarla constante y lealmente siempre que tuviera ocasión de hacerlo. El doctor Ortiz amablemente me invitó entonces a que escribiera unas pocas palabras acerca de mi "conversión" terminológica, y ello fue el motivo de mis presentes párrafos.

En efecto, en el apartado de Contrapunteo titulado “Del fenómeno social de la transculturación’ y de su importancia en Cuba", Ortiz aclara que:

Entendemos que el vocablo transculturación expresa mejor las distintas fases del proceso transitivo de una cultura a otra, porque este no consiste solamente en adquirir una distinta cultura, que es lo que en rigor indica la voz angloamericana acculturation, sino que el proceso implica también necesariamente la pérdida o desarraigo de una cultura precedente, lo que pudiera decirse una parcial desculturación, y, además, significa

\footnotetext{
1 "Pensamiento periférico, redes intelectuales y circulación de ideas", Centro Cultural de la Cooperación, C.A.B.A, 17 de mayo de 2017.

2 Eduardo Devés-Valdés, Pensamiento periférico, Buenos Aires, CLACSO-IDEA-USACH, 2014.

3 Fernando Ortiz, Contrapunteo cubano del tabaco y el azúcar, La Habana, Jesús Montero, [1940] 1991.

${ }_{4}$ Ambos ya habían realizado destacados aportes a las Ciencias Sociales. Ortiz venía publicando desde inicios de siglo una densa obra referida principalmente a los afrocubanos, campo de estudio del cual fue pionero. Malinowski había publicado en 1922 Los argonautas del Pacifico occidental, etnografía seminal de perdurable prestigio.
} 
la consiguiente creación de nuevos fenómenos culturales que pudieran denominarse de neoculturación. [...] En conjunto, el proceso es una transculturación, y este vocablo comprende todas las fases de su parábola [...] Sometido el propuesto neologismo, transculturación, a la autoridad irrecusable de Bronislaw Malinowski, el gran maestro contemporáneo de etnografía y sociología, ha merecido su inmediata aprobación. Con tan eminente padrino, no vacilamos en lanzar el neologismo susodicho. ${ }^{5}$

En primer lugar, tenemos entonces el carácter procesual de idea de transculturación, tal como señaló Melville Herskovits al diferenciar el concepto de difusión, como "estudio de la transmisión cultural conseguida", del de transculturación, como "estudio de la transmisión cultural en marcha". En segundo lugar, está el hecho de que la transculturación incluye diversos sub-procesos: ${ }^{6}$

[...] desculturación o exculturación, para la primera fase histórica de destrucción colonialista; inculturación, como sustitutivo de aculturación, para el fenómeno lineal de sumisión a la cultura de conquista; transculturación, para la fase plena del proceso, esto es, para el intercambio en los dos sentidos; y neoculturación, para la creación y definición de la cultura producto de todo el proceso. $^{7}$

Para el tema de este artículo, es importante saber que el concepto de transculturación fue retomado en Chile por Alejandro Lispchutz, médico e investigador letón que llegó al país en 1926 constituyéndose, desde la década de 1940, en la figura principal de indigenismo local. ${ }^{8}$ El concepto en marras apareció formalmente enunciado en Los últimos fueguinos: transculturación y desculturación, extinción y exterminación. ${ }^{9}$ Este artículo retomó el tema de dos conferencias dictadas en 1960 en la Universidad de Concepción y en el INAH de México, elaboradas en base a su participación en la Misión Científica Chilena para el Estudio del Indio Fueguino de 1946. En línea con la propuesta de Ortiz, ${ }^{10}$ Lipschutz entendía por transculturación a "la transición más o menos brusca de un pueblo, o etnos, de su propia cultura ancestral a la cultura de otro etnos con el cual entró en contacto; por la fuerza de las circunstancias, sea que estuviesen éstas en acuerdo o en pugna con la consciente voluntad del etnos en transculturación". ${ }^{11}$ Allí también Lipschutz describió las diversas posibilidades del proceso: "Un etnos, en contacto con otro etnos", y -sujeto a la transculturación- "abandona ciertos elementos constituyentes de su propio patrimonio cultural" o "los adapta al conjunto del patrimonio cultural que lo ha invadido, o le ha sido impuesto" e "incorpora elementos constituyentes del conjunto cultural ajeno [...] en forma tal cual, o [...] transformándolos en acuerdo con sus necesidades especiales". ${ }^{2}$ El concepto de transculturación ingresó así al universo de Lipschutz y fue puesto en sintonía con diversos desarrollos intelectuales que este venía implementando desde fines de la década de 1930, los cuales trataré a continuación.

\footnotetext{
5 Ortiz, op. cit., p. 90.

${ }^{6}$ Melville Herskovits, El hombre y sus obras. La ciencia de la antropología cultural, México D.F., FCE, [1948] 1974, pp. 565-567.

${ }_{7}^{7}$ Julio Le Riverend, Ortiz y sus contrapunteos, Fernando Ortiz Contrapunteo cubano del tabaco y el azúcar, La Habana, de Ciencias Sociales, [1978] 1991, pp. V-XXXVIII.

8 Bernardo Berdichewsky, Alejandro Lipschutz, su visión indigenista y antropológica, Santiago, UCSH, 2004.

9 Lipschutz, Alejandro, "Los últimos fueguinos: transculturación y desculturación, extinción y exterminación", Genus 18 (1-4), 1962, pp. 89-115.

10 Ortiz, op. cit.

11 Alejandro Lipschutz, "Los últimos fueguinos: transculturación y desculturación, extinción y exterminación”, Genus 18 (1-4), 1962 , p. 89.

12 Ibidem, p. 90.
} 


\section{Resurrección indoamericana y transculturación}

En Americanismo y raza india, Lipschutz discutió "las ideas biológico raciales [que se aplicaban] de modo pseudo científico a fenómenos históricos y sociales para fines ajenos a la ciencia misma, como lo es la propaganda política". ${ }^{13}$ Refutó prejuicios sobre los efectos del mestizaje, la supremacía racial blanca y el degeneramiento biológico de los pueblos originarios, proponiendo en cambio indagar las relaciones de poder históricamente situadas entre dominados y dominadores desde la conquista española hasta el presente. ${ }^{14}$

Retomó los aportes de Bronislaw Malinowski, Paul Rivet, Carl Jung y Hermann Müller, para anotar que las investigaciones del primero habían demostrado la lógica económica y moral que sustentaban las reglas, costumbres y creencias de la vida privada y pública de los pueblos "primitivos"; las del segundo que las diferentes agrupaciones humanas tenían un origen común; las del tercero que el funcionamiento psíquico del hombre "primitivo" era idéntico al del hombre "civilizado"; y las del cuarto que no existía evidencia genética que sustentase las ideas biológico-raciales. Esto le llevó a concluir que el hombre "representa una entidad biológica muy uniforme desde el punto de vista evolutivo, biológico y cultural, a pesar de todo su polimorfismo biológico y cultural". ${ }^{15}$ Para aquilatar debidamente estos postulados, debemos tomar en cuenta su contexto de enunciación, tanto por lo temprano del mismo, como por el apogeo de los proyectos de eugenesia en el mundo.

La "raza india" podía ser en parte caracterizada biológicamente en razón de ciertos rasgos fenotípicos, pero este concepto era realmente aplicable sólo a un grupo social determinado: aquél que había sido dominado desde la conquista y cuya función social había sido servir a las agrupaciones dominantes. Así, "raza india es toda la gran masa popular indígena o mestizada, la que en su mayoría permanece en estado económico, físico y cultural lamentable, por no haber todavía terminado en Indoamérica el período de encomienda". ${ }^{16} \mathrm{El}$ indoamericanismo propuesto era la reivindicación de los derechos económicos y culturales que les habían sido arrebatados a los indígenas por las agrupaciones sociales dominantes, y este incluía la participación de los dominados en la creación de una tradición nacional, la cual sería "la obra creadora espiritual colectiva de un grupo de hombres ligados por intereses comunes vitales en un territorio limitado" la que se podía efectuar "sólo con la incorporación de las masas indígenas como factor activo [...] en la vida de los pueblos hispanoamericanos". ${ }^{17}$ Resurrección indoamericana fue el término con el que tituló la reivindicación de esas masas desheredadas, acción que ayudaría a la clausura del coloniaje económico-cultural al que nuestros países estaban sometidos.

Por otra parte, múltiples razones hacían convergir los intereses de las diferentes naciones hispanoamericanas en un movimiento mayor, el del internacionalismo hispanoamericano: "Si condiciones de orden geográfico, histórico y cultural determinaron y siguen determinando una división nacional entre los pueblos hispanoamericanos, hay otras condiciones que los reúnen en una confederación internacional", entre otras "el idioma común, las condiciones semejantes de estructura económica y social, la semejanza de la composición racial, las vastas posibilidades de desarrollo económico y cultural, al ser realizada la confederación". ${ }^{18}$ De modo que sólo a través de la "reorientación social" de América, hecho

\footnotetext{
13 Alejandro Lipschutz, Indoamericanismo y raza india, Santiago, Nascimento, 1937, p. 15.

14 Tuillan Yuing y Yuri Caravajal, "El efecto filosófico de la obra de Lipschutz", Cuadernos de Pensamiento Latinoamericano 19, 2013, pp. 50-63.

${ }_{15}$ Lipschutz, 1937, op. cit., p. 44, el resaltado del autor.

16 Lipschutz, 1937, op. cit., p. 63.

17 Lipschutz, Ibidem, p. 67.

18 Lipschutz, Ibidem, p. 68.
} 
que implicaba la incorporación de los dominados como agentes activos de transformación, se romperían los lazos de sujeción que impedían el desarrollo de nuestras naciones.

El concepto de transculturación, como he dicho, apareció en Los últimos fueguinos. ${ }^{19}$ Transculturación, en la forma presentada por Lipschutz, tiene íntima relación con el llamado proceso de préstamo cultural de cuño teórico difusionista. Recordemos que en el mismo 1) operaba un proceso selectivo de asimilación de los rasgos que mejor se adaptaban a la cultura receptora, y 2) que esta recepción incluía un proceso de transformación necesario para su mejor adaptación al nuevo entorno social. ${ }^{20}$

El uso del concepto de transculturación como categoría heurística aplicada al desarrollo histórico lleva a proponer que Lipschutz, diferenciándose del sesgo antropológico de la época, no situó a los colectivos indígenas en un presente sincrónico, ni los tomó como unidades independientes. Este entendía a la cultura como un todo constituido por partes interdependientes, elementos materiales y espirituales vitales para la supervivencia y el desarrollo de un grupo humano en su entorno natural mediante los cuales este construía su "tradición orgánica vivida". Desde su perspectiva, la transculturación era un proceso vivido por todos los pueblos en todas las épocas del desarrollo humano. El mismo acontecía al entrar en contacto un grupo con otro/s y suponía el traspaso de elementos culturales entre los mismos en el contexto de las economías que cada grupo desarrollaba. A lo largo de la historia humana se había dado un proceso constante de intercambios culturales, ya sea mediante relaciones pacíficas o violentas, los que llevaron al cambio de la estructura socio-económica de las agrupaciones sociales. Nótese que la transculturación era pensada siempre partiendo a nivel de la estructura: el sistema socio productivo constituye la base del patrimonio cultural, ya que través de sus elementos se realiza la vida del grupo. Así, el mestizaje biológico y cultural no podía ser entendido como algo negativo per se sino como una condición del ser humano. En Americanismo y raza india esta idea ya está presente cuando, al oponerse a las razones de los defensores del degeneramiento racial, se postula que los pueblos "no decaen y no desaparecen por factores biológicos inmanentes, sino por factores de orden social, engendrándose estos los últimos en factores de la economía de ese mismo pueblo o en otros pueblos con los cuales se choca en la superficie de la tierra". ${ }^{21}$

Así, la resurrección indoamericana sería el corolario del proceso de transculturación contemporáneo, uno que no debía ser copia de modelos de desarrollos foráneos, sino que debía darse a partir de las culturas de nuestros propios pueblos, incorporando aquellos valores ajenos que pudieran servirles. Se entendía que las naciones americanas sólo terminarían de formarse con la incorporación económica y espiritual de las masas indígenas, desarrollándose entonces una conciencia cultural sustentada en los aportes de las diferentes agrupaciones sociales. Latinoamérica no concluiría su independencia sin antes generar una tradición nacional autóctona, "obra creadora espiritual colectiva de un grupo de hombres ligados por intereses comunes vitales en un territorio limitado", y esta no podría lograrse sin la incorporación económica y espiritual de las masas indígenas. ${ }^{22}$

\section{Estudios andinos y marxismo en Chile}

El uso a través del tiempo de un mismo concepto supone un proceso de transferencia que incluye tanto apropiación como reelaboración, con su consecuente modificación de sentido. Piénsese cuan diferente

\footnotetext{
${ }^{19}$ Lipschutz, 1962, op. cit.

20 María Carozzi, María Maya y Guillermo Magrassi, Conceptos de antropología social, Buenos Aires, Centro Editor de América Latina, 1980.

${ }^{21}$ Lipschutz, 1937, op. cit., p. 50.

22 Ibidem, p. 41.
} 
es el caso de la sociedad cubana desde la óptica de Ortiz, cuando este advierte que los prejuicios raciales son consecuencia de una desigualdad material entre explotadores y explotados, que el de las colonias imperiales, en donde el funcionalismo malinowskiano exhibe la creencia ilusoria y voluntarista en la homeostasis e integridad cultural de los pueblos sujetos al indirect rule de la Pax Britannica. En el caso de Lipschutz, a su vez, el concepto de transculturación correrá cercano al propuesto por Ortiz, ya que el problema indígena es pensado siempre en clave histórica y a través de la relación entre agrupaciones sociales dominantes y dominadas, como sucede desde sus obras tempranas. Visto de este modo, resulta lógico asumir que el significado de cualquier concepto debe ser aprehendido "en acción" y no en un imaginado estado puro, por fuera del campo social, del contexto en el que este gravita. En razón de lo anterior, en lo siguiente realizaré una breve descripción de un conjunto de científicos sociales marxistas interesados en problemáticas andinas para, en el próximo apartado, analizar la incidencia del concepto de transculturación y de ciertas ideas antitéticas de Mariátegui en un debate extraído de la producción textual originada en el marco del I Congreso del Hombre Andino.

En su análisis de la relación entre políticas estatales e investigación social sobre aymaras y atacameños en el norte de Chile, Hans Gundermann y Héctor González señalan que, entre 1960 e inicios de 1970, diversos profesionales fueron convocados a "diagnosticar o informar los alcances, las consecuencias e impacto" de políticas como la reforma agraria. Y también que "un grupo de cientistas sociales se planteará objetivos más radicales como el del tránsito de la comunidad andina al socialismo". ${ }^{23}$ Los investigadores inscritos en esta última línea fueron Ximena Aranda, Freddy Taberna, Lautaro Núñez, Patricio Núñez y Gabriel Martínez.

\begin{abstract}
Algunos de estos jóvenes profesionales, a su vez, tenían una formación marxista previa y se nutren ideológicamente del contacto con intelectuales nacionales como el arqueólogo Julio Montané o la figura de Alejandro Lipschutz, además de nexos con otros intelectuales de izquierda en Perú, Bolivia y Argentina. Sus conocimientos sobre el mundo andino regional provenían de sus viajes por las zonas interiores, de su participación en algún proyecto específico [...] y del conocimiento de iniciativas [institucionales] desarrolladas en la zona $[\ldots]^{24}$
\end{abstract}

Lo anterior permite esbozar una incipiente red de cientistas interesados por problemáticas andinas que se fortaleció no sólo por sus labores académicas sino también por el avance de la izquierda y -más aúnpor la implementación de la vía chilena al socialismo durante la presidencia de Allende. Gran parte de estos mismos investigadores llevaron adelante y/o estuvieron presentes en el referido I Congreso del Hombre Andino. Ampliaré entonces los datos anteriores.

La investigadora Ximena Aranda Baeza (Santiago, 1932), egresó de la Universidad de Chile y se especializó luego en Geografía Humana en la Universidad de Burdeos. Participó del Instituto de Geografía de la Universidad de Chile, realizando desde 1960 estudios sobre estructura agraria. Estuvo a cargo de la macro zona norte de la Oficina Regional de Planificación (ORPLAN) entre 1970 y 1973, con la llegada de la Unidad Popular al poder.

Freddy Taberna fue un geógrafo formado en el Pedagógico de la Universidad de Chile. Hacia fines de los sesentas dirigió, acompañado por Lautaro Núñez y el alcalde de Iquique, Jorge Soria Quiroga, el "Plan Andino" dedicado al etnodesarrollo aymara. Esta experiencia sirvió de base para trabajos sobre religiosidad andina y geografía tarapaqueña. Durante el gobierno de Allende fue Director Regional de la

${ }^{23}$ Hans Gundermann y Héctor González, "Sociedades indígenas y conocimiento antropológico. Aymaras y atacameños de los siglos XIX y XX”, Chungara 41 (1), 2009, p. 117.

${ }^{24}$ Ibidem, p. 118. 
Oficina de Planificación Nacional (ODEPLAN) con sede en Iquique, profesor de la cátedra de Geografía en la Universidad de Chile Zona Norte y secretario regional del Partido Socialista. Fue fusilado en 1973 en Pisagua. Gundermann y González plantean que había estrecho contacto entre Taberna y Aranda, se entiende que por razones de orientación disciplinar y labor de gestión, además de las ideológicas que estoy tratando. 25

Lautaro Núñez es un arqueólogo egresado también del Pedagógico de la Chile en 1962. En 1963 se hizo cargo del museo de Calama de la misma Universidad. Allí dirigió el Programa Arqueología y Museos entre 1963 y 1974, en el marco del cual se practicaba etnografía. ${ }^{26}$ Lo anterior llevó a tomar contacto al equipo con la problemática aymara, hecho que impulsó el diseño del Plan Andino anteriormente citado. ${ }^{27}$ Sabemos que entre Núñez y Taberna estos contactos iban más allá de lo académico y la política, ya que eran iquiqueños, del mismo barrio (El Morro) y se llevaban pocos años de edad. ${ }^{28}$ En el Programa Arqueología y Museos revistió el hermano de Lautaro, el también arqueólogo Patricio Núñez. Ambos estaban ligados a la corriente de inspiración marxista denominada Arqueología Social Latinoamericana y -por lo tanto- a Julio Montané29 y Felipe Bate. Esta corriente se constituyó con el concurso de los arqueólogos que, orientados por el materialismo histórico, entendieron a su labor como un aporte a la conformación de una arqueología de orientación más social y comprometida con el proceso sociopolítico coetáneo. ${ }^{30}$

El matrimonio de antropólogos conformado por Gabriel Martínez y Verónica Cereceda llegó desde Bolivia en 1971 en donde trabajaban con comunidades aymaras. Núñez rememora que inmediatamente tomó contacto con ellos para incorporarlos a la Universidad de Chile en Iquique. En 1972 esta Universidad firmó un convenio con el Servicio Estatal de Cooperación Técnica, designándose a Martínez como encargado del programa de artesanía aymara con base en Isluga. Los esposos se sumaron luego a la Universidad del Norte de Iquique, en donde crearon el Centro Isluga de Investigaciones Andinas. ${ }^{31}$ El nudo básico de relaciones se completa con Alejandro Lipschutz. Más allá del lógico conocimiento que Núñez -en razón del alcance de su figura- tenía sobre el trabajo de Lipschutz, existían contactos personales. La relación de amistad se extendía también al discípulo de Lipschutz, Bernardo Berdichewsky, ${ }^{32}$ antropólogo interesado en la problemática indígena, quien resume en su apreciación de la misma lo más granado de la tradición lipschutziana:

El problema del campesinado indígena de Latinoamérica ha sido, fundamentalmente -desde el momento en que se instituyó el sistema de la hacienda- un problema de superexplotación económica y social, en cuanto fuerza de trabajo agrícola, la que ha permitido también producir la renta de la tierra para la clase terrateniente. Pero, en su caso, estuvo acompañado además por la discriminación racial, la que servía como máscara de la explotación social y económica y como uno de los elementos

\footnotetext{
25 Ibidem.

26 Lautaro Núñez, Carta a Alejandro Lipschutz, Iquique, 1967.

27 Lautaro Núñez, Sobre los comienzos de los estudios andinos y sus avances actuales en el norte de Chile, Carlos Zanolli, Julia Costilla, Dolores Estruch y Alejandra Raamos (comps.), Los estudios andinos hoy. Práctica intelectual y estrategias de investigación, Rosario, Prohistoria, 2013, pp. 79-122.

28 Lautaro Núñez, Freddy Taberna Gallegos: del Morro a la leyenda iquiqueña. Bernardo Guerrero (ed.) Homenaje a Freddy Taberna G. Escritos sobre la sociedad aymara del Norte Grande de Chile, Iquique, CREAR-El jote errante, 1996, pp. 9-16.

${ }^{29}$ Durante la dictadura Montané se exilió en México, en donde fue acogido por el Instituto Nacional de Antropología e Historia, institución a cargo de Guillermo Bonfil Batalla. Sus fundamentos para una arqueología marxista pueden verse en Marxismo y Arqueología, entre otras obras.

30 Andrés Troncoso, Diego Salazar y Donald Jackson, "Ciencia, Estado y Sociedad: retrospectiva crítica de la arqueología chilena”, Arqueología sudamericana 4 (2), 2008, pp. 122-145.

31 Núñez, op. cit., 2013

32 Lautaro Núñez, op. cit., 1967.
} 
superestructurales, ideológicos, que permitían reproducir el sistema. Por eso no es suficiente para el análisis de la situación social del campesinado indígena tratar, exclusivamente, las variables socio-económicas y clasistas y dejar de lado los aspectos étnicos, los que, en realidad, están íntimamente relacionados con aquéllos. ${ }^{33}$

La vinculación entre Núñez y Lipschutz sucedía al menos desde fines de la década de 1960, ya que se preservan cartas cruzadas entre ambos en el Fondo Lipschutz de la Universidad de Chile. Por ejemplo, esta de Núñez fechada en Iquique el 16 de noviembre de 1967:

He recibido su atenta carta en los momentos de mi trabajo de campo. Ud. bien sabe cuánto se debe el arqueólogo al terreno para crear, por fin, las problemáticas y metodologías que tanto necesitamos en Latinoamérica. Por supuesto que debo felicitarlo por el ciclo de conferencias del Instituto Indigenista, piense cuanto nos falta para difundir los valores indígenas en nuestro país, ahogado por mistificaciones de los siempre listos historiadores clasistas. [...] He revisado recientemente más de veinte comunidades mestizas, de tipo Aymara, ubicadas en las cercanías de la frontera Chileno-Boliviana que corresponde a la misma unidad étnica altiplánica. Allí he observado grupos humanos, totalmente aislados que necesitan con urgencia estudios antropológicos. Mi papel de arqueólogo-antropólogo me lleva sólo a tomar muchos datos que mañana servirán al especialista además que anoto todas las supervivencias prehispánicas en estas comunidades actuales como un medio de interpretar mejor el pasado arqueológico.

Retengamos la segunda parte de la cita, ya que expresa acabadamente la visión predominante sobre la problemática indígena que sostendría la Comisión Organizadora del I Congreso del Hombre Andino, tal como se desprende del Simposio 4, "El rol de la sociedad andina y el tránsito al socialismo". ${ }^{34}$ En la correspondencia queda clara la admiración de Núñez por Lipschutz, del que se consideraba "discípulo". ${ }^{5}$ Esto llevaría a proponerle al "notable indigenista" que brindara la conferencia inaugural del referido Congreso "para los efectos de otorgarle un marco mayor". 36

En el apartado final delinearé los puntos principales de la conferencia inaugural de Lipschutz ${ }^{37}$ y abundaré en la fundamentación del Simposio $4 .{ }^{38}$ Esto me permitirá ponderar la incidencia de Mariátegui y Lipschutz en estos jóvenes investigadores que realizaron estudios pioneros en donde i) se ponía en foco la crisis del campesinado andino y la escasa respuesta de los entes estatales y de las universidades;39 ii) se criticaba la "aplicación de técnicas e instrumentos ajenos a nuestra realidad y, por lo tanto, difíciles de aplicar dadas las diferencias que existen en nuestro medio y aquellos países donde estas técnicas han sido

\footnotetext{
33 Bernardo Berdichewsky, Etnicidad y clase social en los mapuches, 1979, disponible en: www.blest.eu/cs/berdichewsky.html (24/12/2015).

34 Freddy Taberna Gallegos y Comisión Organizadora del I CHA, Simposio No 4: El rol de la sociedad andina y el tránsito al socialismo. Informe: Algunas Condiciones Básicas para el estudio del tránsito hacia el Socialismo de la Sociedad Andina, Bernardo Guerrero (ed.), Homenaje a Freddy Taberna G. Escritos sobre la sociedad aymara del norte grande de Chile, Iquique, CREAR-El Jote Errante, [1973] 1996, pp. 47-65.

35 Núñez, 1967, op. cit.

36 Núñez, 2013, op. cit., p. 94.

37 Alejandro Lipschutz, El próximo futuro de los pueblos indígenas andinos: problemas fundamentales, discurso inaugural del ICHA, Universidad de Chile (ed.), I Congreso del Hombre Andino. Antofagasta, Universidad de Chile, 1973, 4 pp.

38 Taberna Gallegos y Comisión Organizadora, op. cit.

39 v.g. Lautaro Núñez, La crisis del campesinado andino regional, Oficina de Planificación Nacional, Macro-Zona Norte, Antofagasta, 1972.
} 
elaboradas"; 40 y iii) se proponía romper con el enfoque tecnicista, a través de una mirada centrada en la relación del hombre y su medio, debidamente contextualizada y en perspectiva histórica. ${ }^{41}$

\section{El I Congreso del Hombre Andino}

\section{Sobre el Congreso}

El I Congreso del Hombre Andino sesionó en Arica, Iquique y Antofagasta en junio de 1973. Fue organizado por la Universidad de Chile y auspiciado por la Dirección de Cultura de la Presidencia de la República, la Sociedad Chilena de Arqueología y Sociedad Chilena de Antropología (Universidad de Chile 1973a). El Congreso venía siendo ideado desde comienzos de la presidencia de Allende, ya que fue promocionado en el Seminario Internacional Proceso de Integración Andina (Arica 1971), evento patrocinado por el Consejo de Rectores de las Universidades Chilenas y la Junta de Adelanto de Arica, orientado a problemáticas contemporáneas y a "entender y aplicar el Pacto Andino como un desafío posible para unir más a las universidades del área". ${ }^{42}$

La Comisión Organizadora estaba compuesta por investigadores ya mencionados, entre ellos Julio Montané, Lautaro Núñez, Patricio Núñez y Freddy Taberna. ${ }^{43}$ Su visión sobre el etnodesarrollo, vinculada a la vía chilena al socialismo y a los procesos de descolonización, quedó expresada en el informe "Algunas Condiciones Básicas para el estudio del Tránsito hacia el Socialismo de la Sociedad Andina". ${ }^{44}$ En este sentido, Lautaro Núñez, presidente del Congreso, expresó que la integración andina comenzaba "con la unidad de sus hombres en luchas comunes, en este caso es la búsqueda de un camino común para interpretar y ubicar la sociedad andina bajo el vigor de las ciencias sociales". 45

El Congreso fue "un evento de carácter panandino, internacional e interdisciplinario (...) el último encuentro masivo de arqueólogos y cientistas sociales" antes de que se interrumpiese el orden democrático. ${ }^{46}$ En el mismo "se hizo patente la dificultad para el diálogo en el ambiente general. [john] Murra no pudo hablar porque los estudiantes no lo permitieron. Y Gabriel Martínez tampoco, porque tenía un tema que no era relevante políticamente (...) las artesanías aymara". ${ }^{47}$ En el mismo sentido, Oscar Espoueys opina que "en el momento del Congreso del '73 habíamos perdido la capacidad para el diálogo, la recuperamos al reencontramos en Salta [en el III Congreso Nacional de Arqueología Argentina de 1974]". ${ }^{48}$ Calogero Santoro apunta que en el evento

no sólo se discutió acerca de los logros del "hombre" andino con sus proyecciones y significados en el presente, sino también cómo las soluciones andinas habían alcanzado hasta los inhóspitos parajes del desierto de Atacama. Pero, además [...] se enfrentaron perspectivas contrapuestas, reflejo del complicado ambiente político del

\footnotetext{
40 Freddy Taberna Gallegos, Los Andes y el altiplano tarapaqueños, una tentativa de evaluación geográfica, Santiago, Universidad de Chile, 1968.

41 v.g. Ximena Aranda, Rafael Barahona y René Saá, Elementos diagnósticos para un plan de desarrollo local en San Pedro de Atacama, Santiago, CORFO-IG/UCH, [1961] 1968.

42 Núñez, 2013, op. cit. p. 87.

43 Núñez, 1996, op. cit.

44 Freddy Taberna y Comisión Organizadora, op. cit.

45 Lautaro Núñez, Introducción, Universidad de Chile, Boletín Especial I Congreso del Hombre Andino. 20 al 30 junio de 1973, 1973a, $\mathrm{s} / \mathrm{p}$.

46 Sociedad Chilena de Arqueología Boletín de la Sociedad Chilena de Arqueología, Año 12, Número Especial Punta de Tralca, 1995 , p. 13.

47 Sociedad Chilena de Arqueología, Ibídem, p. 18.

48 Sociedad Chilena de Arqueología, Ídem, la aclaración es mía.
} 
país y del continente en general, a tal punto que el Congreso fue interrumpido en Antofagasta por el 'tanquetazo', un intento anticipatorio de las fuerzas armadas chilenas por derrocar al gobierno socialista de Salvador Allende. ${ }^{49}$

El I Congreso del Hombre Andino operó como un espacio en donde confluyeron múltiples perspectivas y en donde se dieron a conocer los diferentes avances alrededor del desarrollo de las sociedades andinas desde sus orígenes a la actualidad. ${ }^{50}$ Sobre esta base, el evento intentó evaluar, alrededor de las problemáticas actuales de las comunidades andinas "su marginalidad, la desintegración de la matriz económica-cultural y los efectos de la modernidad y del Estado para compartir inclusivamente estrategias a través de un diálogo franco ante la diversidad del Centro Sur Andino". ${ }^{51}$ Lautaro Núñez recuerda que en el Congreso "surgieron temas arqueológicos, antropológico-sociales y principalmente etnohistóricos". 52 Pese a que al momento de su realización se contaba con las fuentes financiamiento necesarias, ${ }^{53}$ las actas definitivas no pudieron ser publicadas a raíz de la intervención militar a las universidades, aunque sí lo fueron algunos resúmenes (en un Boletín Especial que salió de imprenta antes de realizarse el Congreso) y los informes de temas para los simposios. ${ }^{54}$

\title{
Conferencia de Lipschutz
}

Los aportes de Lipschutz que van desde 1940 a 1960 se inscriben en lo que podría denominarse una primera etapa de su pensamiento, de impronta asimilacionista y fuertemente ligada a las premisas del Instituto Indigenista Interamericano. Su segundo momento intelectual, que se extiende desde finales de 1960 hasta su fallecimiento en 1980 queda bien condensado en la conferencia del I Congreso del Hombre Andino, cuyo eje central se resume en la siguiente cita:

\begin{abstract}
He pensado que para todos nosotros sería útil que, en primer lugar, nos diéramos cuenta de la razón por la cual estamos todos muy ocupados, y aún preocupados, del problema del "hombre Andino". Y, por otra parte, es muy grato para mí y para todos ustedes dejar constancia que el Programa del Congreso elaborado por la Comisión Organizadora evidencia sabiduría: no se trata de un problema sino de un gran conjunto de problemas que son tanto de interés puramente científico, o teórico, como de un interés práctico y de importancia suma [...] Al inaugurar este I CONGRESO DEL HOMBRE ANDINO conviene prestar nuestro interés a algunos otros problemas básicos para el Hombre Andino y los indios todos. Me refiero al conjunto de problemas que emanan de los complejos sociales que pueden ser resumidos con los términos "Ley de la Tribu", "Ley de la Gran Nación" de la cual las tribus forman parte y "Ley del Patriotismo Doble" que de esta situación social forzosamente emana. Es un gran conjunto de problemas que deberían ser céntricos en todo Congreso Americanista. ${ }^{55}$
\end{abstract}

En efecto, a partir de las incipientes demandas de grupos regionales y étnicos en todo el mundo y del ocaso del indigenismo y de las políticas indigenistas asimilacionistas, Lipschutz venía profundizando en

${ }^{49}$ Calogero Santoro, "Las conversaciones siguen a pesar del ruido. Dedicado a John V. Murra". Chungara, Revista de Antropología Chilena 42 (1), 2010, pp. 83-86.

50 Lautaro Núñez, "De las apariciones y andanzas de John Murra por el desierto de Atacama y como construyó su misión innovadora", Chungara 42 (1), 2010, p. 132.

51 Núñez, 2013, op. cit., p. 94-95.

52 Ibidem, p. 94.

53 Núñez, 1973a, op. cit.

54 Universidad de Chile, Informes de temas para los simposios del Primer Congreso del Hombre Andino. Antofagasta, Universidad de Chile, 1973a. Universidad de Chile, Resúmenes de ponencias presentados. Antofagasta, Universidad de Chile, 1973b.

55 Alejando Lipschutz, 1973, op. cit. 
la defensa del derecho de los pueblos indígenas a la autonomía. Visibilizando su presencia cuando ésta se encontraba marginada en la mayoría de los países, intuyó que la reemergencia de la conciencia identitaria y de los valores culturales de los grupos minoritarios era síntoma de la persistencia dentro de las naciones de lo que llamó tribalismo. E insistió en que la revisibilización de esos grupos debería dar lugar al reconocimiento de su autonomía cultural y territorial en el marco de la "gran nación" hispanoamericana. Esta idea tomaba el modelo original de la URSS, que preveía la creación de repúblicas federadas en las cuales se daría una doble pertenencia: a la propia república y a la unión de repúblicas. Ese "patriotismo doble" podría nacer también en Hispanoamérica si los pueblos originarios obtenían autonomía cultural. ${ }^{6}$ En tanto la autonomía de tales pueblos no era una realidad ajena a los procesos de transformación que nuestras sociedades habían experimentado a lo largo de la historia, esta podía formar lícitamente parte de las alternativas a futuro.

$\mathrm{Al}$ abogar por la formación de una nueva unidad político-territorial en la que se confederasen los pueblos y las naciones, basando su idea de nación en un internacionalismo indoamericano que reconociese la diversidad cultural, dejaba planteada la posibilidad de una reorganización de las naciones que incluyese la autonomía territorial de los pueblos indígenas. ${ }^{57}$ Lo cual constituye la característica más marcada de esta segunda y última etapa intelectual, la del indianismo autonomista, el cual constituye el mensaje fundamental de su conferencia.

\section{El Simposio 4}

La fundamentación del Simposio 4 del Congreso se titula "El rol de la sociedad andina y el tránsito al socialismo". Informe: "Algunas Condiciones Básicas para el estudio del tránsito hacia el Socialismo de la Sociedad Andina" y fue firmada por Freddy Taberna y por la Comisión Organizadora. El documento en sí es mucho más de lo que hoy entendemos por fundamentación de una mesa o simposio, ya que constituye una evaluación completa de la problemática indígena andina, enmarcada en el contexto latinoamericano y mundial. El Informe consta de dos partes, la primera trata las condiciones que presentaba el campesinado andino, mientras que la parte segunda se interesaba por el campesinado andino chileno en particular. Su objetivo general es comprender la inserción de este actor social en los procesos de transformaciones sociopolíticas coetáneos. El Informe está profundamente influido por las ideas de Mariátegui, tal como trataré en las Conclusiones.

En la primera parte se propone que el desarrollo del proceso histórico regional había generado una ideología cultural propia de las sociedades andinas que era esencialmente anticapitalista. Es lo que se denomina "matriz cultural socialista". Sin embargo, el capitalismo había modificado en parte esta ideología por medio del accionar de las instituciones "burguesas". El análisis del proceso histórico que lleva desde la comunidad primitiva a la sociedad clasista a través del colonialismo español y del capitalismo incipiente de la etapa republicana indicaba que: 1) la conquista representó un choque económico-social y cultural que diezmó muchas instituciones indígenas; 2) la democracia republicana creó un falso concepto de libertad y de igualdad que permitió la pérdida de la tierra indígena y el incremento del latifundio y 3) el proceso avanzó hasta el momento actual, desintegrando, despojando e incorporando al mundo urbano el campesinado andino, bajo los intereses de la burguesía y del capitalismo nacional e internacional.

\footnotetext{
56 Arauco Chihuailaf, "Ley de la tribu, ley de la gran nación y la autonomía de los pueblos indígenas en el pensamiento de Alejandro Lipschutz", Pandora 11, 2012, pp. 107-118.

57 Roberto Morales, El legado teórico a la antropología acerca de los pueblos originarios de Chile, A.A./V.V. Alejandro Lipschutz: nueva antologia, Santiago, ICAL, 2005, pp. 42-111.
} 
El problema fundamental en el área andina era la lucha de clases. Entre los explotados se contaban la masa campesina y los campesinos proletarizados a consecuencia de los procesos migratorios hacia las zonas industriales. La ideología capitalista escondía esta cuestión de fondo al negar la contradicción utilizando términos como "indios" o "mestizos". La ideología desarrollista -que tendía a modernizar el sistema de explotación- resultaba un apoyo a las imposiciones del capitalismo. Por lo tanto, los procesos revolucionarios y/o reformistas en los que el área andina estaba involucrada debían apuntar a la liberación de la sociedad campesina de los medios de explotación de la burguesía terrateniente, del capitalismo monopólico y del imperialismo.

La estructura clasista se sostenía fundamentalmente en el problema agrario. La modificación del sistema tradicional de tenencia comunitaria en favor de la propiedad individual conllevaba la desintegración de la comunidad andina, presa de la servidumbre en un contexto feudal, obstaculizando el relacionamiento de la masa campesina con el proletariado, actor que poseía mayor conciencia de clase. El problema podía solucionarse con la implementación de una reforma agraria de características revolucionarias que, al socializar la tenencia de la tierra, eliminase los intereses de la burguesía terrateniente que buscaban destruir a las comunidades, privar a los campesinos de la tierra y favorecer el latifundio.

Sin desdeñar la condición objetiva primordial de que todos los hombres andinos eran explotados por el capitalismo, se advertía que las sociedades campesinas tradicionales presentaban diferentes relaciones con el medio urbano, diferentes matices culturales y estaban insertas en Estados nacionales cuyos gobiernos presentaban a su vez contrastes. Por lo tanto, se preveía que los hombres andinos participarían de modos diferentes en los procesos de transformaciones sociopolíticas. Con vistas a poder diagnosticar el modo en que estos se involucrarían en el proceso revolucionario, era necesario conocer las demás condiciones que hacían a la diferenciación planteada.

En conclusión, de su mayor comunicación, de su mayor organización laboral y política y de la comprensión del campesinado indígena de su papel en la lucha de clases dependía la posibilidad de quebrar los actuales medios de explotación capitalistas e incorporarse a un modo de producción más afín con su propio desarrollo cultural: el socialista. Ahora bien, aunque la diferencia fundamental se daba entre explotadores y explotados, los autores consideraban que no se podían aplicar sin más las técnicas revolucionarias del medio urbano en el medio rural. En este sentido, la investigación social permitiría conocer mejor al mundo andino, su rol en el proceso revolucionario, las relaciones posibles entre la vanguardia proletaria y el campesinado y la forma en que los partidos populares integrarían a las masas campesinas cuando estas comprendiesen que la supervivencia de su matriz cultural y de su propio desarrollo socio-económico dependían de su incorporación al proceso revolucionario.

La parte segunda del Informe trata las condiciones particulares que presentaba el campesinado en Chile con el objeto de comprender su posible papel en el proceso sociopolítico del país.

En primer lugar, se indicaba que este había sido reiteradamente despojado de su tierra y obligado a participar de la explotación minera e industrial. Lo anterior había llevado a su deterioro socio-económico, a la alteración de su organización social y de sus pautas culturales y a la dependencia a los intereses de la burguesía regional, estado de cosas que podía denominarse como interrupción del desarrollo histórico andino. Se denunciaba que las políticas públicas no habían mejorado la calidad de vida de los campesinos andinos. Esto podía notarse en el avance del proceso de privatización de la tierra que traía como consecuencia una baja de la producción agrícola y la pérdida de la organización laboral colectivista en una región de características ecológicas desérticas, que se mantenía gracias a un delicado equilibrio; en la pérdida demográfica de los hábitats andinos como consecuencia de la migración hacia los centros urbanos 
y minero-industriales regionales; y en la penetración de instituciones públicas que habían impuesto los intereses capitalistas en la educación, el comercio, la seguridad y el régimen agrícola.

La supremacía de la institucionalidad burguesa por sobre la de los pueblos andinos se hacía evidente también en el pluripartidismo del sistema democrático burgués, el cual falseaba los fines políticos de las comunidades, orientados por los viejos moldes socio-políticos autóctonos. En este marco, el sistema político dominante intentaba resolver la desintegración andina mediante tácticas desarrollistas y paternalistas, pero no atacaba la contradicción clasista en tanto esta constituía la posibilidad de su propia existencia. El tipo de relaciones entre las instituciones burguesas y los pueblos andinos marcaba la necesidad de disminuir la explotación ejercida sobre el mundo rural desde los centros urbanos. En adelante, las políticas públicas deberían ajustarse al patrón cultural andino, en un intento de "andinizar" los procesos de cambio haciendo participar a las comunidades en una modificación de las estructuras que posibilitase la implementación de la revolución socialista.

Se señalaba que, si bien el ascenso de la Unidad Popular al poder pudo darse en virtud de circunstancias coyunturales, como la división del electorado de centro-derecha, esta respondía a la vez a un proceso de antigua data vinculado a la tradición democrática chilena y a la temprana conciencia de clase del proletariado, el cual se organizó para enfrentar al capitalismo incipiente. En 1973, el momento de la gestión de Allende era problemático, ya que las medidas que buscaban favorecer a las clases explotadas eran constantemente frenadas por los poderes Legislativo y Judicial, la oligarquía nacional y el imperialismo transnacional. La crisis económica en curso respondía en parte a los desajustes propios del proceso de transición, pero a la vez al accionar de estos actores. Sin embargo, la vía chilena había conseguido logros importantes: nacionalización del cobre y de las riquezas básicas, profundización de la Reforma Agraria y liquidación de los monopolios.

Se aclaraba que ciertas diferenciaciones existentes al interior de la sociedad andina no se fundamentaban en elementos culturales y sociales sino en las desiguales relaciones entre los campesinos y la acumulación de capital impuesta por la burguesía comercial e industrial que manejaba la relación rural-urbana. Esto podía verse en el aumento de la fortuna de ciertos individuos andinos mediante el comercio, lo que los relacionaba con elementos de la burguesía. Sin embargo, el enriquecimiento individual no había llegado a producir clases al interior de las comunidades, sino que se expresaba en fenómenos tales como relaciones paternalistas en las festividades religiosas. La matriz andina, por lo tanto, seguía operando como factor de integración.

Se pensaba que el pragmatismo político, rasgo sobresaliente de las comunidades campesinas, evidenciado en la habilidad de elegir dirigentes que simpatizaban con el signo político del gobierno de turno, no era un rasgo de madurez sino una solución para conservar y aumentar los beneficios materiales. Los líderes adoptaban una posición de izquierda intuitiva sin una posición de clase en tanto no estaban ligados a los intereses del capitalismo, pero, al entrar en juego con la institucionalidad burguesa, adquirían deformaciones que dificultaban el acceso de su sociedad a las transformaciones en marcha y, por otra parte, desconocían la metodología para incorporarse a la situación política contingente. Se advertía que, aunque el hombre andino no comprendía su rol político en el proceso chileno, este estaba más ligado a él que el proletariado en virtud de la supervivencia de la forma comunitaria para definir los problemas básicos de la comunidad, el uso de la propiedad colectiva y los sistemas transhumánticos que permitían la explotación múltiple de regiones situadas más allá de las fronteras nacionales. La vieja matriz cultural en donde estos modos de vida se insertaban se reactivaba cíclicamente en razón del carácter agropecuario de la economía, lo que la ataba irreversiblemente a las transformaciones socio-políticas de la nación. Estos modos de vida y esta matriz cultural debían ser objeto de estudios particulares. 
Las minorías campesinas debían encarar un proceso de liberación o de revolución cultural para dejar de ser dependientes de las pautas culturales de la burguesía. En este trance, la investigación sobre las pautas culturales propias del campesinado era indispensable para rescatarlo y revalorizarlo, ayudando a crear las condiciones para su revolución cultural y la incorporación de la cultura andina socialista al proceso revolucionario. Se advertía que la metodología de estudio había sido hasta ahora implementada a partir de las posiciones teóricas de los centros de dominación mundial. Las investigaciones producidas de esta forma no ponían a las comunidades andinas en el contexto global del desarrollo del capitalismo y -por lo tanto- apoyaban los criterios reformistas-desarrollistas que negaban la lucha de clases. Era necesario entonces que las nuevas investigaciones se realizasen desde las propias concepciones e intereses de la sociedad andina a través de un método materialista dialéctico que pudiera dar razón del desarrollo de las fuerzas productivas que habían determinado la crisis regional. Se entendía que la teoría marxista serviría para analizar la particular relación entre estructura y superestructura de la sociedad andina, en donde los elementos religiosos constituían el impulso dinamizador de la economía y que podían, por lo tanto, ser capaces de agilizar las transformaciones revolucionarias si estas surgían de las propias comunidades.

Finalmente, se aclaraba que la vía chilena al socialismo buscaba preparar agentes que ayudasen a implementar un cambio gradual entre la economía precapitalista de las sociedades andinas y el socialismo. En este marco, la investigación social adquiría un carácter central, ya que se debía conocer en profundidad a los pueblos andinos para que ellos pudiesen lograr sus transformaciones dentro del contexto nacional. Esta también era imprescindible para programar la educación que debería impartirse en las comunidades andinas, lo que colaboraría en la toma de conciencia por parte de estas de su rol histórico y presente. Tanto la nueva legislación (Ley indígena $\mathrm{N}^{\circ}$ 17.729) como el accionar de las instituciones gubernamentales (v.g. ORPLAN, Instituto de Desarrollo Indígena), de las Universidades y programas de estudio (v.g. Plan Andino) habían puesto en foco la crisis contemporánea de los pueblos andinos. A partir de estas experiencias, se necesitaba planificar el desarrollo de los mismos por dentro del proceso revolucionario y en el marco de una discusión activa con estos pueblos.

Pasando en limpio, el Informe proponía que la investigación social debía abocarse a estudiar i) el desarrollo del proceso histórico regional, ii) la particular relación entre estructura y superestructura de la sociedad andina, de la cual se desprendía el mantenimiento de su modo tradicional de vida, iii) las condiciones objetivas generales (explotación) y particulares (sus variadas características culturales) del campesinado andino; iv) las relaciones entre las instituciones burguesas y los pueblos andinos, medio por el cual el capitalismo penetraba y desintegraba la sociedad andina; v) el rol del mundo andino en el proceso revolucionario, las relaciones posibles entre la vanguardia proletaria y el campesinado y la forma en que los partidos populares integrarían a las masas campesinas; vi) la forma en la que se darían las condiciones subjetivas para la incorporación del campesinado al proceso revolucionario cuando estas comprendiesen que la supervivencia de su matriz cultural y de su propio desarrollo socio-económico dependían de su inscripción en el proceso revolucionario. En este contexto, la relevancia de la investigación científica se centraba en su papel político, ya que la misma -al estudiar las condiciones objetivas ("conocer en profundidad") de los pueblos andinos- ayudaría a generar las condiciones subjetivas ("revolución cultural" o toma de conciencia por parte de estos de su rol histórico y presente) que era la segunda precondición para que los mismos pudiesen lograr sus transformaciones dentro del contexto nacional.

Sobre la base de lo anterior quedaba por definir cómo realizar la tarea. El Informe rechazaba de plano toda investigación que estudiase las particularidades de las sociedades andinas sin tomar en cuenta el proceso de desarrollo histórico regional en el marco del sistema mundial capitalista. Esta perspectiva, al negar implícitamente la lucha de clases inherente al proceso, apoyaba las medidas desarrollistas de corte reformista que no conducían a un cambio social revolucionario. Era necesario entonces que las investigaciones se realizasen a partir de las propias concepciones e intereses de los pueblos andinos a 
través de un método materialista dialéctico que pudiese dar cuenta del desarrollo de las fuerzas productivas que estaba en la base de la crisis regional.

\section{Conclusiones}

En estas conclusiones resumiré, en primer lugar, los principales acuerdos entre Mariátegui y Lipschutz en torno al "problema indígena". En segundo lugar, me detendré en dónde sus derroteros se bifurcan, lo cual tendrá implicancia en la evaluación del posible futuro de los pueblos indígenas en el marco del proceso chileno. Estos pasos me permitirán revisar, en tercer y último lugar, la incidencia de ambos pensadores en los fundamentos del Simposio 4 del I Congreso del Hombre Andino.

\section{El "problema indígena"}

Aunque ambos autores parten de campos del indigenismo particulares (político-literario en Mariátegui, político-científico en Lipschutz), ambos sostienen una lectura marxista de la problemática. Existe, además, un claro acoplamiento de Lipschutz a las ideas del peruano, quien lo precede temporalmente. Así como Mariátegui señaló que "Las razas indígenas se encuentran en la América Latina en un estado clamoroso de atraso y de ignorancia, por la servidumbre que pesa sobre ellas, desde la conquista española", ${ }^{58}$ Lipschutz -en forma similar- apuntó que la "raza india [...] en su mayoría permanece en estado económico, físico y cultural lamentable, por no haber todavía terminado en Indoamérica el período de encomienda". ${ }^{9}$ Conviene recordar que en Mariátegui la categoría de raza refiere simultáneamente a las características biológicas y a la historia civilizacional particulares de un grupo humano, ${ }^{60}$ mientras que, para Lipschutz, ${ }^{61}$ aunque existen ciertas características fenotípicas comunes, raza india hace referencia preferentemente a una agrupación social dominada desde la conquista y cuya función social había sido servir a las agrupaciones dominantes.

De lo anterior se desprende que el "problema del indio" es de tipo económico-social y que "arranca de nuestra economía. Tiene sus raíces en el régimen de propiedad de la tierra", por lo que su solución "tiene que ser una solución social [y] sus realizadores deben ser los propios indios", ${ }^{62}$ propuesta que seguirá sin dilaciones Lipschutz. ${ }^{63}$ Planteado el problema, se abre el espacio para la proposición de un plan de acción, centrado en las categorías de socialismo indoamericano ${ }^{64}$ y de indoamericanismo. ${ }^{65}$

Dice Mariátegui “No queremos, ciertamente, que el socialismo sea en América calco y copia. Debe ser creación heroica. Tenemos que dar vida, con nuestra propia realidad, en nuestro propio lenguaje, al socialismo indo-americano". ${ }^{66} \mathrm{El}$ peruano buscaba vincular así la revalorización social, política y cultural de los pueblos indígenas con la perspectiva de la revolución socialista, y -al mismo tiempo- indicar la importancia de las tradiciones económicas colectivistas de las comunidades indígenas para el desarrollo del socialismo en la región. Por su parte, el indoamericanismo propuesto por Lipschutz era la

\footnotetext{
58 José Mariátegui, El problema de las razas en la América Latina, Ideología y política, José Mariátegui, Mariátegui total: 100 años, Lima, Amauta, [1929] 1994.

${ }^{59}$ Lipschutz, 1937, op. cit., p. 63.

60 Aníbal Quijano, Cuestiones y horizontes: de la dependencia histórico-estructural a la colonialidad / descolonialidad del poder, Buenos Aires, CLACSO, 2014.

${ }^{61}$ Lipschutz, 1937, op. cit.

62 José Mariátegui, José Siete ensayos de interpretación de la realidad peruana, Lima, Minerva, [1928] 2007, pp. 35 y 49.

63 Lipschutz, 1937, op. cit.

${ }^{64}$ Mariátegui, [1928] 2007, op. cit.

65 Lipschutz, 1937, op. cit.

${ }^{66}$ Mariátegui, [1929] 1994, op. cit. p. 249.
} 
reivindicación de los derechos económicos y culturales de los indígenas que les habían sido arrebatados por las agrupaciones sociales dominantes, y este incluía la participación de los dominados en la creación de una tradición nacional, la cual sería "la obra creadora espiritual colectiva de un grupo de hombres ligados por intereses comunes vitales en un territorio limitado" la que se podía efectuar "sólo con la incorporación de las masas indígenas como factor activo [...] en la vida de los pueblos hispanoamericanos. Sólo así llegará a su término feliz el proceso formativo de las nuevas naciones indoamericanas". 67

\section{Desacuerdos}

Como queda señalado, existía una comunidad muy fuerte de ideas entre ambos autores. ¿En dónde residían las diferencias, entonces? Creo que hay dos puntos a tratar: en primer lugar, una de orden práctico, referida a sus posicionamientos sobre la autonomía política indígena y, en segundo lugar, otra de orden teórico, centrada en la categoría de transculturación. Si la opresión era un asunto de clase, de raza o de nacionalidad fue un tema que se debatió en la Conferencia Comunista Latinoamericana realizada en Buenos Aires en 1929. Allí Mariátegui propuso soluciones prácticas al problema indígena: expropiar los latifundios serranos en favor de las comunidades; transformar a las comunidades en cooperativas de producción; apoyar la lucha de los yanaconas contra los hacendados para eliminar la institución parasitaria del enganche; educar ideológicamente a las masas indígenas. ${ }^{68}$ Sin embargo, esta defensa no lo llevó a apoyar la formación de una república indígena entre los pueblos quechua y aymara, tema que se discutió particularmente en ese evento. Él creía que esta medida no conduciría a la adopción del socialismo entre los indígenas, sino a la conformación de otro estado burgués, con todas las contradicciones internas y externas de los mismos, concluyendo que sólo una revolución socialista que incluyese a las masas indígenas explotadas podría permitirles a estas incorporar el sentido de la liberación, posibilitándose así su autodeterminación política. ${ }^{69}$

Lipschutz, ${ }^{70}$ por su parte, creía firmemente en lo que denominó reorientación social del continente latinoamericano, no especificando si pensada como cambio revolucionario o reformista, por medio de las armas o de las urnas. Lo que es claro es que esta reorientación debía incluir a las masas indígenas como elemento activo y, además, que su sistema ideal era uno que integrase distintos tipos de bloques autónomos. De allí lo de Ley de La Tribu, Ley de la Gran Nación y Ley del Patriotismo Doble. En su parecer, la República de Chile podría tomar la forma de lo que hoy denominamos un Estado plurinacional, el cual incluyese diversas autonomías étnicas. A diferencia de lo propuesto por Mariátegui, para el área andina señaló en particular la posibilidad de una República Autónoma Quechua y una República Autónoma Aymara. ${ }^{71} \mathrm{La}$ amalgama de la compleja estructura política estaría dada por la necesaria doble pertenencia a ambos sistemas políticos. A su vez, Lipschutz presuponía una unión tácita entre las naciones americanas antiguamente colonizadas por España. En este sentido, pensaba que entre estas se podría formar una confederación y que sólo así llegaría "a su término feliz el proceso formativo de las nuevas naciones indoamericanas". ${ }^{72}$

Queda finalmente realizar una consideración sobre el desarrollo del proceso histórico americano. Como vengo tratando, existía en principio acuerdo entre ambos autores sobre los efectos de la conquista

\footnotetext{
${ }^{67}$ Lipschutz, 1937, op. cit., p. 67.

68 Mariátegui, [1929] 1994, op. cit.

${ }^{69}$ Marc Becker, "Mariátegui y el problema de las razas en América latina", Revista andina 35, 2002, pp. 191-220.

${ }^{70}$ Lipschutz, 1937, op. cit.

${ }^{71}$ Ídem.

${ }^{72}$ Ibidem, p. 67.
} 
española y la situación indígena durante la colonia y la república. A lo largo del tiempo se habían conformado naciones signadas por las relaciones entre un grupo dominante y otro dominado, estando incorporadas en el segundo las poblaciones originarias. Estas naciones, a su vez, se hallaban sometidas al poder imperialista. Frente a un panorama tan desalentador, un factor importante tanto de la perduración como de la incorporación de los indígenas al proceso de cambio deseado era su estructura social de carácter colectivista.

Ahora bien, la gran diferencia que encuentro entre ambos pensadores se centra en que para Mariátegui la liberación indígena -y su misma subsistencia, en suma- dependía del triunfo de la revolución socialista. En cuanto a Lipschutz, su énfasis en el proceso de transculturación le permitía oponerse a los que entendían que la incorporación de los elementos llamados occidentales destruiría a la llamada "cultura indígena". Por el contrario, bajo determinadas circunstancias este fenómeno podía incluso estimular su renacimiento. ${ }^{73}$ Sin embargo, también alertó que ciertas modificaciones nocivas habían causado el deterioro de las comunidades indígenas a raíz de la estrecha interdependencia entre los componentes culturales. ${ }^{74}$ La lectura de Lipschutz, entonces, dejaba abierta la posibilidad de diferentes desenlaces y esto resultaba de entender a la transculturación como un proceso no teleológico.

\section{Mariátegui y Lipschutz en el Simposio 4}

A la hora de pensar la incidencia de los autores indigenistas en el Informe del Simposio 4, vale indicar que este no cuenta con otra bibliografía que tres citas textuales de Mariátegui. ${ }^{75}$ Como queda claro, los autores toman como punto de partida el planteamiento central de los Siete Ensayos consistente en que el problema indígena es de tipo económico-social, cosa avalada también por Lipschutz. A partir del mismo podemos entender la insistencia con borrar del texto la categoría "indígena" y reemplazarla por otras homólogas entre sí: sociedad andina, campesinado andino, hombre andino, sociedad campesina tradicional, masa campesina y campesinos proletarizados, campesinado indígena, pueblos andinos y comunidades campesinas, que sirven tanto para situar a estos grupos sociales en un determinado medio (el andino) como para caracterizarlos según su actividad económica (campesinado) y colocarlos dentro de la clase explotada. La negación a utilizar categorías como "indio" o "mestizo" debe leerse en el sentido propuesto coetáneamente por Bonfil Batalla, ${ }^{76}$ para quien el "indio" era una "categoría supraétnica producto del sistema colonial, y sólo como tal puede entenderse". Por un lado, esto es un rechazo explícito a la forma de abordaje estructural funcionalista, en tanto se entendía que su pretendida apoliticidad conllevaba implícitamente un posicionamiento reaccionario. Esta crítica adquiere una claridad meridiana cuando se trata la discriminación a la que se hallan sometidos los pueblos originarios. Los autores entienden que esta se daba en razón de mantener al campesinado en una posición dominada para favorecer el sostenimiento del statu quo.

Ya he señalado que, aunque el Informe enfatice el estado contemporáneo del campesinado en el marco de las relaciones capitalistas, plantea también la necesidad de realizar un estudio del desarrollo histórico regional. La conquista española es señalada como un choque económico-cultural que impactó sobre las instituciones indígenas. Esta fue el embrión del futuro desarrollo del sistema capitalista, ya que implementó un régimen social basado en las relaciones entre dos agrupaciones sociales: los dominadores

\footnotetext{
73 Chihuailaf, op. cit.

74 Morales, op. cit.

75 Mariátegui, [1928] 2007, op. cit.

${ }^{76}$ Guillermo Bonfil Batalla, "El concepto de indio en América: una categoría de la situación colonial", Anales de Antropología Revista del instituto de Investigaciones Antropológicas de México 9, 1972, p. 117.
} 
y los dominados, las cuales subsistían en la actualidad y a las que el texto alude bajo las categorías marxistas de explotadores y explotados, que hacen hincapié en la base económica de tal dominación.

En este sentido, se señala que la investigación científica debía indagar las particularidades de los pueblos originarios incluidas en su común matriz cultural, partiendo del reconocimiento de las condiciones objetivas generales (la oposición clasista explotadores-explotados) con la finalidad de ayudar a que se concretasen las condiciones subjetivas necesarias para la incorporación del campesinado a la revolución socialista, en sus propios términos y desde sus propias concepciones. ¿A qué aludían los autores con esto último? Debemos pensar aquí en las experiencias etnográficas que se venían implementando, como la del Plan Andino. Lautaro Núñez rememora que en la concepción del mismo “[...] prevaleció la idea de que inicialmente Freddy debía empaparse de esa realidad, recorriendo pueblo a pueblo y así llegar a una proposición que recogiera las iniciativas de las propias comunidades". ${ }^{77}$

Se trataba entonces de integrar las particulares concepciones del desarrollo indígena que no se opusieran a los fundamentos socialistas -tarea al parecer no improbable en tanto la propia matriz cultural de las comunidades era de carácter comunitaria- y de ayudar a realizar la modificación gradual de las instituciones de los pueblos originarios que no estuvieran en consonancia con estos fundamentos. En esencia, el cambio relatado era el del recorrido desde una economía precapitalista, contaminada por la injerencia del capitalismo, hacia una economía socialista. Este podía entenderse como una vuelta potenciada y mejorada a los principios rectores de la matriz cultural del campesinado andino.

Ahora bien, nótese que -al poner en primer plano la poca diferenciación interna de las comunidades- el Informe relega el hecho de que el capitalismo tiene efectos globales y -por lo tanto- todo grupo humano está inserto en mayor o menor medida en su red. Además, ata a la sociedad campesina a una "tradición" poco propensa al cambio en virtud de su base económica agropecuaria de carácter cíclico. En este contexto, el capitalismo aparece como el agente iniciador del cambio social, aunque este produzca efectos degenerativos. Cuando los autores tratan las modificaciones producidas en el tráfico caravanero, desde los viejos patrones transhumánticos que permitían el aprovechamiento colectivo de una vasta red de recursos, hasta la actual utilización del mismo para el tráfico de estupefacientes, se entiende que lo hacen con el objeto de ejemplificar los efectos perniciosos de las nuevas necesidades impuestas por el capitalismo. Pero erran en no reconocer la capacidad adaptativa de estos actores a los múltiples escenarios históricos y el papel que juega el acaparamiento de bienes materiales en las relaciones intra e interétnicas.

Es mi parecer que en el Informe aparece también la influencia de Lispchutz. Por ejemplo, cuando se trata la necesaria emergencia de las condiciones subjetivas que harán que los pueblos originarios se sumen a las transformaciones en curso, luego de tomar conciencia de su rol histórico. Aparece aquí el término "revolución cultural" como grado superior de conciencia al cual la investigación marxista está llamada a hacer un gran aporte. Sostengo que este concepto es solidario del de resurrección indoamericana, forma con la que Lispchutz retrató la reivindicación económica y cultural de los indígenas. Ambas denominaciones aluden al término de la desintegración de las comunidades andinas y al inicio de la revitalización de las mismas en el marco del proceso revolucionario que Lipschutz entendió como una futura y necesaria reorientación social del continente americano.

Por otra parte, aunque el análisis hecho en este artículo induzca a pensar que la característica eminente del Informe, su objeto político, lleva a aplicar un tanto esquemáticamente los conceptos marxistas, en uno de sus puntos se resalta un interesante aspecto que lo desliga de la aplicación mecánica de la teoría marxista. En efecto, cuando se propone investigar la particular relación entre estructura y superestructura

77 Núñez, 1996, op. cit., p. 12. 
de la sociedad andina, lo que se quiere indicar es que la religión opera como motor de la actividad económica, ya que las festividades dan inicio y fin al ciclo agropecuario. No significa esto que la base material deje de determinar en última instancia a los componentes superestructurales, sino que -en el modo de producción de estas sociedades- la ritualidad domina las relaciones entre los diferentes integrantes del ayllu, cosa que establece una taxativa diferencia con la sociedad capitalista. En efecto, los ayllus -unidad de parentesco básico- son el marco en el cual las unidades domésticas cooperan entre sí y, por lo tanto, el medio a través del cual la estructura económica se activa mediante principios de reciprocidad vehiculizados por prácticas rituales. En este sentido, sería correcto el planteo de los autores en torno a complejizar la relación entre base y superestructura; pero debe advertirse que el dominio de la estructura económica no estaría operado desde la religión misma sino desde las relaciones sociales a través de las cuales cualquier ideología se organiza y, además, que estas mismas relaciones se hallan imbricadas en los dos órdenes del modo de producción.

Para finalizar, visto que en el Informe pueden encontrarse influencias de ambos autores, vale preguntarse si existe un predominio de las ideas de Mariátegui o de las de Lipschutz. Pese al gran respeto de la Comisión Organizadora del I Congreso del Hombre Andino por la figura del segundo, cosa que se deduce de su invitación para brindar la conferencia inaugural, creo que es lícito pensar que los jóvenes andinólogos encontraban mayor arraigo en las proposiciones de Mariátegui. Puede proponerse que, desde la perspectiva de los autores, si el proceso de transculturación planteado por Lipschutz arrojaba un resultado seguro era el de la pronta desaparición de las comunidades indígenas, de no triunfar en lo inmediato sobre la reacción la vía chilena de la Unidad Popular.

Ahora bien, cualquier cuestionamiento actual no debería ignorar, como señalaron Gundermann y González "el sentido de inclusión social que implicaba la propuesta' [...] pese al exceso ideológico y su voluntarismo". ${ }^{78} \mathrm{~A}$ la luz de la historia posterior, que incluyó los procesos de reemergencia étnicas de colectivos étnicos como aymaras y atacameños, creo finalmente que el caso en marras puede ayudarnos a pensar la particular y cambiante relación entre los tiempos históricos y los enfoques político-académicos, en Chile en particular y en Suramérica en general.

\section{Bibliografía}

Aranda, Ximena; Barahona, Rafael y Saá, René, Elementos diagnósticos para un plan de desarrollo local en San Pedro de Atacama, Santiago, CORFO-IG/UCH, [1961] 1964.

Becker, Marc, “"Mariátegui y el problema de las razas en América latina”, Revista andina 35, 2002, pp. 191 220.

Berdichewsky, Bernardo, Alejandro Lipschutr, su visión indigenista y antropológica. Santiago, UCSH, 2004.

Bonfil Batalla, Guillermo, "El concepto de indio en América: una categoría de la situación colonial", Anales de Antropología Revista del instituto de Investigaciones Antropológicas de México 9, 1972, pp. 105-124.

Carozzi, María; Maya, María y Magrassi, Guillermo, Conceptos de antropología social, Buenos Aires, Centro Editor de América Latina, 1980.

Chihuailaf, Arauco, "Ley de la tribu, ley de la gran nación y la autonomía de los pueblos indígenas en el pensamiento de Alejandro Lipschutz", Pandora 11, 2012, pp. 107-118

Devés-Valdés, Eduardo, Pensamiento periférico. Buenos Aires, CLACSO-IDEA-USACH, 2014.

Gundermann, Hans y González, Héctor, "Sociedades indígenas y conocimiento antropológico. Aymaras y atacameños de los siglos XIX y XX”, Chungara 41 (1), 2009, pp. 113-164.

Herskovits, Melville, El hombre y sus obras. La ciencia de la antropología cultural, México D.F., FCE, [1948] 1974.

78 Gundermann y González, op. cit., p. 118. 
Le Riverend, Julio, "Ortiz y sus contrapunteos", Fernando Ortiz, Contrapunteo cubano del tabaco y el azúcar, La Habana, de Ciencias Sociales, [1978] 1991, pp. V-XXXVIII.

Lipschutz, Alejandro, Americanismo y raza india. Santiago, Nascimento, 1937.

Lipschutz, Alejandro, "Los últimos fueguinos: transculturación y desculturación, extinción y exterminación", Genus 18 (1-4), 1962, pp. 89-115.

Lipschutz, Alejandro, "El próximo futuro de los pueblos indígenas andinos: problemas fundamentales, discurso inaugural del ICHA", Universidad de Chile I Congreso del Hombre Andino, Antofagasta, Universidad de Chile, 1973.

Malinowski, Bronislaw, "Introducción", Fernando Ortiz, Contrapunteo cubano del tabaco y el azúcar, La Habana, de Ciencias Sociales, [1940] 1991, p. XXXI-XXXVIII.

Mariátegui, José Siete ensayos de interpretación de la realidad peruana, Lima, Minerva, [1928] 2007.

Mariátegui, José, "El problema de las razas en la América Latina, Ideología y política", José Mariátegui, Mariátegui total: 100 años, Lima, Amauta, [1929] 1994, pp. XX.

Morales, Roberto, "El legado teórico a la antropología acerca de los pueblos originarios de Chile", A.A./V.V., Alejandro Lipschutz: nueva antología: 42-111, Santiago, ICAL, 2005, pp. 402-411.

Núñez, Lautaro, La crisis del campesinado andino regional, Oficina de Planificación Nacional, Macro-Zona Norte, Antofagasta, 1972.

Núñez, Lautaro, "Introducción”, Universidad de Chile, Boletín Especial I Congreso del Hombre Andino. 20 al 30 junio de 1973, 1973a, p. 1.

Núñez, Lautaro, "Simposio 2: Verticalidad y colonización andina pre-europea", Universidad de Chile $I$ Congreso del Hombre Andino, 1973b, 1 p.

Núñez, Lautaro, "Freddy Taberna Gallegos: del Morro a la leyenda iquiqueña”. Bernardo Guerrero (ed.),

Homenaje a Freddy Taberna G. Escritos sobre la sociedad aymara del Norte Grande de Chile, Iquique, CREAR-El jote errante, 1996, pp. 9-16.

Núñez, Lautaro, "De las apariciones y andanzas de John Murra por el desierto de Atacama y como construyó su misión innovadora", Chungara 42 (1), 2010, pp. 127-139.

Núñez, Lautaro, "Sobre los comienzos de los estudios andinos y sus avances actuales en el norte de Chile", Carlos Zanolli, Julia Costilla, Dolores Estruch y Alejandra Ramos (comps.) Los estudios andinos hoy.

Práctica intelectual y estrategias de investigación, Rosario, Prohistoria, 2013, pp. 79-122.

Ortiz, Fernando, Contrapunteo cubano del tabaco y el azúcar, La Habana, de Ciencias Sociales, [1940] 1991.

Santoro, Calogero, "Las conversaciones siguen a pesar del ruido. Dedicado a John V. Murra", Chungara 42 (1), 2010, pp. 83-86.

Sociedad Chilena de Arqueología, Boletín de la Sociedad Chilena de Arqueología, Año 12, Número Especial Punta de Tralca, 1995.

Quijano, Aníbal, Cuestiones y horizontes: de la dependencia bistórico-estructural a la colonialidad/ descolonialidad del poder, Buenos Aires, CLACSO, 2014.

Santoro, Calogero, "Las conversaciones siguen a pesar del ruido. Dedicado a John V. Murra", Chungara 42 (1), 2010, pp. 83-86.

Taberna Gallegos, Freddy, Los Andes y el altiplano tarapaqueños, una tentativa de evaluación geográfica, Santiago, Universidad de Chile, 1968.

Taberna Gallegos, Freddy y Comisión Organizadora, "Simposio $N^{\circ}$ 4: El rol de la sociedad andina y el tránsito al socialismo. Informe: Algunas Condiciones Básicas para el estudio del tránsito hacia el Socialismo de la Sociedad Andina", Bernardo Guerrero (ed.), Homenaje a Freddy Taberna G. Escritos sobre la sociedad aymara del norte grande de Chile, Iquique, CREAR-El Jote Errante, [1973] 1996, pp. 47-65.

Troncoso, Andrés; Salazar, Diego y Jackson, Donald, Ciencia, Estado y Sociedad: retrospectiva crítica de la arqueología chilena, Arqueología sudamericana 4 (2), 2008, pp. 122-145

Universidad de Chile, Informes de temas para los simposios del Primer Congreso del Hombre Andino, Antofagasta, Universidad de Chile, 1973a.

Universidad de Chile, Resúmenes de ponencias presentados, Antofagasta, Universidad de Chile, 1973b. 
Yuing, Tuillan y Caravajal, Yuri, "El efecto filosófico de la obra de Lipschutz”, Cuadernos de Pensamiento Latinoamericano 19, 2013, pp. 50-63.

Berdichewsky, Bernardo, Etnicidad y clase social en los mapuches, 1979, disponible en: http://www.blest.eu/cs/berdichewsky.html (24/12/2015).

Núñez, Lautaro, Carta a Alejandro Lipschutz, Iquique, 1967. Fondo Lipschutz-Universidad de Chile. 